\title{
Preliminary Study on the Magnetic Properties of GeMn Nanocolumn/Ge Multilayers
}

\author{
Thi Giang Le ${ }^{1 *}$, Minh Tuan Dau ${ }^{2}$ \\ ${ }^{1}$ Department of Engineering and Technology, Hong Duc University, Thanh Hóa, Vietnam \\ ${ }^{2}$ Interdisciplinary Center of Nanoscience of Marseille (CINaM-CNRS), Aix-Marseille University, Campus of Luminy, Marseille, \\ France \\ Email: ^lethigiang@hdu.edu.vn
}

How to cite this paper: Le, T.G. and Dau, M.T. (2020) Preliminary Study on the Magnetic Properties of GeMn Nanocolumn/Ge Multilayers. Materials Sciences and Applications, 11, 441-449.

https://doi.org/10.4236/msa.2020.117030

Received: May 18, 2020

Accepted: July 13, 2020

Published: July 16, 2020

Copyright $\odot 2020$ by author(s) and Scientific Research Publishing Inc. This work is licensed under the Creative Commons Attribution International License (CC BY 4.0).

http://creativecommons.org/licenses/by/4.0/

\begin{abstract}
$\mathrm{Ge}_{0.94} \mathrm{Mn}_{0.06}$ nanocolumn thin film is a unique phase of GeMn diluted magnetic semiconductors (DMS) which exhibit Curie temperature $\left(\mathrm{T}_{\mathrm{C}}\right)>400 \mathrm{~K}$. The multilayers of $\mathrm{Ge}_{0.94} \mathrm{Mn}_{0.06}$ nanocolumns separated by nano-scaled spacers represent great interests for spintronic applications, such as spin valves or giant magneto-resistance (GMR) multilayers. In this article, we present the results obtained from the preliminary study on the exchange coupling in two types of GeMn nanocolumn/Ge multilayers. All the samples have been grown using molecular beam epitaxy (MBE). The superconducting quantum interference device (SQUID) magnetometer has been used to determine the magnetic properties of the samples. In the multilayer system $\mathrm{Ge} /\left[\mathrm{Ge}_{0.94} \mathrm{Mn}_{0.06}(40\right.$ $\mathrm{nm}) / \mathrm{Ge}(\mathrm{d} \mathrm{nm})]_{9} / \mathrm{Ge}_{0.94} \mathrm{Mn}_{0.06}(40 \mathrm{~nm}) / \mathrm{Ge}$, no exchange coupling can be observed. Inversely, exchange coupling between the layers exists and depends on the thickness of the Ge spacers for the GeMn nanocolumns/Ge multilayer spin valve systems. The exchange coupling in the nanocolumns multilayer systems has been shown to be complex due to the leakage field induced by neighboring nanocolumns and the magnetic anisotropy of nanocolumns.
\end{abstract}

\section{Keywords}

Multilayers, Ferromagnetic Semiconductor, GeMn Nanocolumns, Exchange Coupling, Thin Film

\section{Introduction}

The discovery of giant magneto-resistance (GMR) in metallic multilayers by A. Fert and P. Grunberg opened the door to a new field of science [1] [2]. The study of magnetic coupling in multilayer systems is a very specific subject, which often requires a correlation between experimental results and complex theoretical 
models. The applications from this study are numerous and mainly focused on the fabrication of magneto-resistive components and magneto-optics. In the study of the exchange coupling between ferromagnetic layers, the separating layer (latter called spacer) plays a very important role, because the nature, the length and the energy of the coupling strongly depend on this one. Most studies in this field have focused on the coupling between ferromagnetic layers through a non-magnetic and generally metallic layer. For this type of spacer, several theoretical models have been suggested in order to explain the origin of the exchange coupling when it exists [3] [4] [5]. Concerning semiconductor spacers, the study of exchange coupling remains very limited due to the problems related to diffusion at interfaces and to alloy formation (metal/semiconductor systems) or to limited magnetic properties as in the case of magnetic semiconductor/systems. Previous studies have shown the initially positive results in the GaMnAs/GaAs/GaMnAs superlattice [6], in the $\mathrm{Fe} / \mathrm{Si} / \mathrm{Fe}$ or the Co/Si systems [7] [8] [9], and in the stacking of PbS/EuS layers [10]. All these studies show that the multilayers provided with a semiconductor spacer have an FM or AF exchange coupling and that the switching between these two configurations is completely possible. However, the major drawback which could limit their development in several fields of applications comes from their low Curie temperature as well as problems related to the growth and the crystalline quality of the ferromagnetic layers and the spacer.

It has been shown that the interdiffusion between the ferromagnetic layer and the spacer in metallic multilayer systems constitutes a constraint major [11]-[17]. This problem is known as the main cause of the decrease in performance of all systems. To overcome these obstacles, the synthesis of DMS-based multilayers is an attractive approach, because these materials are much more flexible than metals, particularly with regard to integration into conventional CMOS technology based on $\mathrm{Si}$ and Ge. Among numerous phases of GeMn DMS, GeMn nanocolumns appears to be the most interesting, because it is a unique phase exhibiting Curie temperature higher than $400 \mathrm{~K}$ and epitaxial growing on the $\mathrm{Ge}$ substrates [18] [19] [20] [21]. Therefore, the synthesis of a multilayer system based on high Curie temperature GeMn nanocolumns is proof of the emergence of a new material generation intended for the multilayer spintronics. Particularly, by using real time control approach, we have been able to produce 10 stacked layers of GeMn nanocolumns free of $\mathrm{Mn}_{5} \mathrm{Ge}_{3}$ clusters, which are separated by a nano-scaled Ge spacer layer [22] [23]. However, the evidence of an exchange coupling in this system remains to be proven, because no study was published at the time of writing this manuscript. In this paper, we preliminarily study the coupling in GeMn nanocolumns/Ge multilayers and will explain in a very simple and rather intuitive way the first magnetic results obtained.

\section{Experimental}

GeMn film growth was performed using solid source molecular beam epitaxy on 
epi-ready $n$-type $\mathrm{Ge}(001)$ wafers with a nominal resistivity of $10 \Omega \cdot \mathrm{cm}$. The base pressure in MBE is better than $1 \times 10^{-10}$ Torr. The deposition chamber is equipped with reflection high energy electronic diffraction (RHEED) to monitor the epitaxial growth process. Germanium powder $\geq 99.999 \%$ and Manganese powder $99.99 \%$ were evaporated using standard Knudsen effusion cells. The cleaning of the Ge surfaces was carried out in two steps: a chemical cleaning and an in situ thermal desorption of the surface oxide. After this step, a 40-nm-thick Ge buffer layer was grown at $250^{\circ} \mathrm{C}$ to ensure a good starting surface. Next, using real-time control approach, the $\mathrm{Ge}_{1-\mathrm{x}} \mathrm{Mn}_{\mathrm{x}}$ films were subsequently grown at the temperature of $130^{\circ} \mathrm{C}$ and $\mathrm{Mn}$ concentrations of $\sim 6 \% \mathrm{Mn}$ for all layers. The $\mathrm{Ge}_{0.94} \mathrm{Mn}_{0.06}$ layers were separated by the Ge spacer of several nanometers. The magnetic properties were characterized using superconducting quantum interference device (SQUID) magnetometer in the temperature range from 5 to $400 \mathrm{~K}$ with a magnetic field applied parallel or perpendicular to the surface of the samples. The diamagnetic contributions due to the Ge substrate were subtracted from the measurements, leaving the magnetic contributions of the GeMn films.

\section{Results and Discussion}

\subsection{Study the Exchange Coupling in the Multilayer System $\mathrm{Ge} /\left[\mathrm{Ge}_{0.94} \mathrm{Mn}_{0.06}(40 \mathrm{~nm}) / \mathrm{Ge}(\mathrm{d} \mathrm{nm})\right]_{9} / \mathrm{Ge}_{0.94} \mathbf{M n}_{0.06}(40 \mathrm{~nm}) / \mathrm{Ge}$}

Conventional multilayer systems for studying exchange coupling consist of a stack of several ferromagnetic layers spaced apart by non-magnetic layers, the thickness of which is variable. For GeMn multilayers, the ferromagnetic layers of $40 \mathrm{~nm}$-thick were chosen, which ensures good crystalline quality and a homogeneous distribution of the nanocolumns in the layers. A series of 5 period samples has been developed with a wide range of Ge thicknesses ( $d=6,8,15 \mathrm{~nm}$ ). The samples were encapsulated by a layer of Ge 2 - $3 \mathrm{~nm}$ thick. The TEM images of the samples have been shown in Figure 2 and Figure 3 of the ref. 22.

In Figure 1, we present the hysteresis cycles of these three samples measured with the external magnetic field parallel to the sample plane (parallel configuration). Although the rectangular shapes of the cycles reflect a ferromagnetic behavior of all the layers, some interesting points should be underlined. The coercive fields and the residual magnetizations practically did not change whereas the saturation magnetizations were obviously modified. The saturation fields $\mathrm{H}_{S}$ defined as the fields for which the magnetization reaches $95 \%$ of its saturation value, meanwhile, have the same values for all the samples. This last remark is very important because the energies necessary to saturate three samples will have the same value, which means that the thickness of the spacer has no effect on the saturation of the magnetic moments of the layers. We cannot conclude that there is an exchange coupling in this multilayer architecture. However, it is not excluded that there is exchange coupling between the layers (FM type). In this case, the coupling field $\mathrm{H}_{\mathrm{ex}}$ should be sufficiently small so that the reversing field can switch the two magnetizations at the same time. It therefore seems necessary 
to probe still other thicknesses of Ge in order to have a more definitive conclusion.

We also present in Figure 2 the magnetic measurements of these three samples in the perpendicular configuration. We can see the same coercive field values of the saturation field for the three samples and the normalized curves are identical. This means that no coupling can be observed for this configuration.

\subsection{Study the Exchange Coupling in Spin Valve Type Structures}

For the applications in devices using the MRG effect such as spin valves, it is desirable that a bilayer system be made up of a ferromagnetic layer having a greater coercive field than that of the other layer. The layer with the greatest coercive field is called the hard layer and retains the orientation of its magnetization when the external field is varied while we observe a tilting of the magnetization

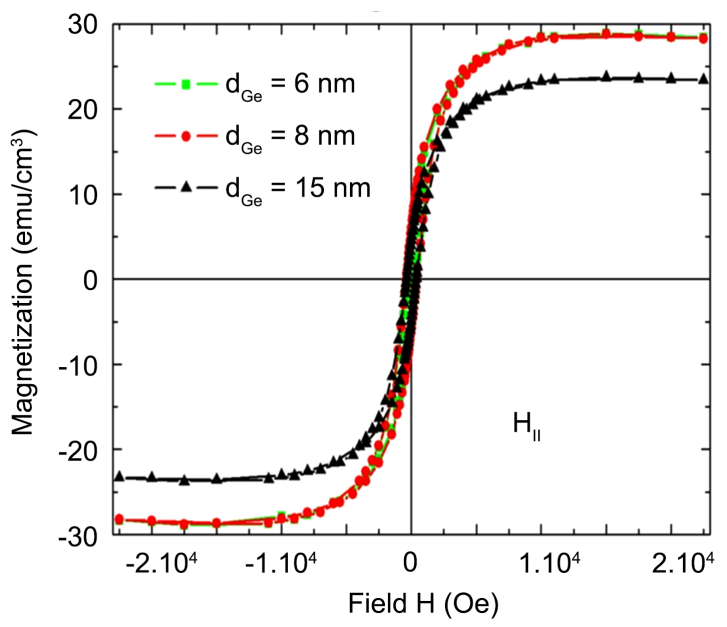

(a)

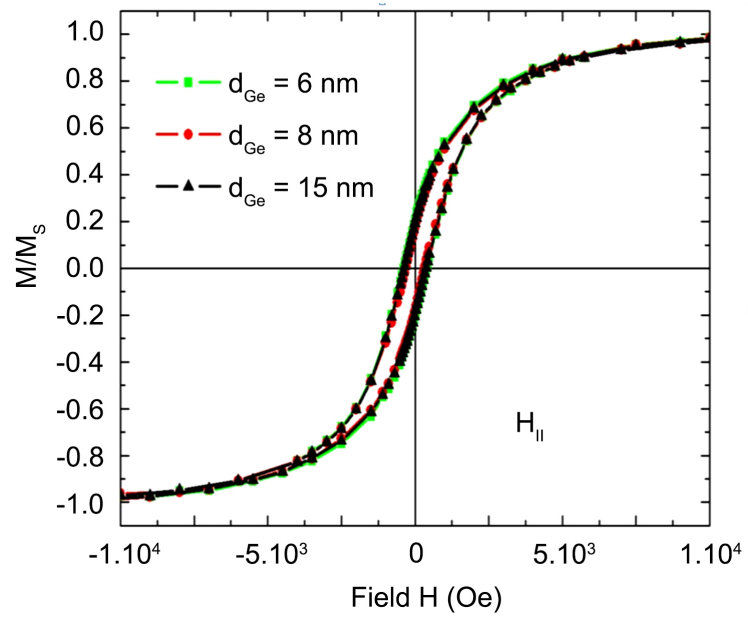

(b)

Figure 1. Hysteresis curves measured at $10 \mathrm{~K}$ of three samples with different Ge spacer layer thicknesses (a) and Superimposition of the 3 normalized curves on the same graph, confirming no exchange coupling is visible by measuring the hysteresis cycles for this range of Ge thicknesses (b). 


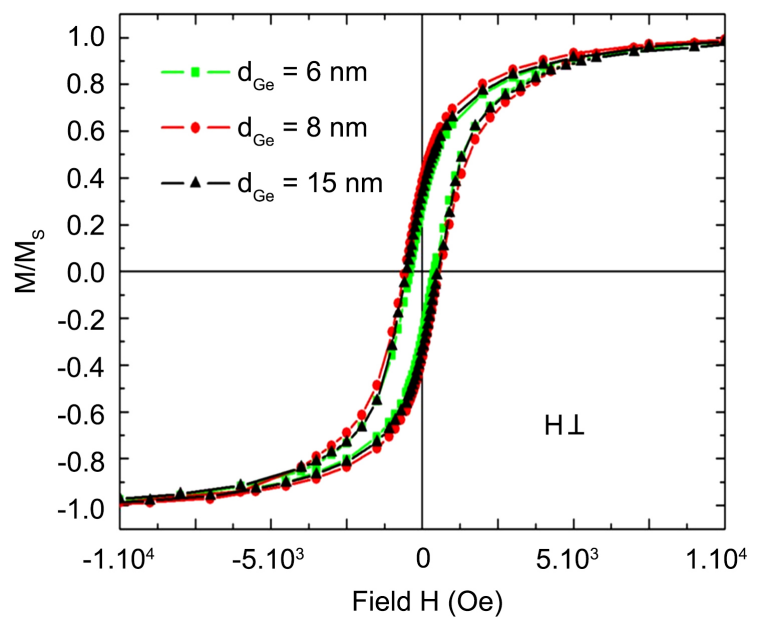

Figure 2. Superimposition of hysteresis curves with a perpendicular external field.

of the less rigid layer (soft layer) if the coupling FM exchange is not strong enough. In the case of nanocolumns, we are trying to modify the coercivity of one layer by increasing its thickness compared to the other. A series of three different thicknesses $d$ of the $\operatorname{GeMn}(45 \mathrm{~nm}) / \mathrm{Ge}(\mathrm{d}) / \mathrm{GeMn}(25 \mathrm{~nm})$ spacer has been grown to study the exchange coupling between the ferromagnetic layers. All the samples are protected by a thin layer of $3 \mathrm{~nm}$ of Ge in order to avoid the oxidation problem which can alter the results such as, for example, by generating an exchange shift AF/FM (exchange bias) [24].

We present in Figure 3(a) the hysteresis cycles of three samples at three $d$ values measured at $\mathrm{T}=10 \mathrm{~K}$ with a magnetic field applied parallel to the sample (the TEM images of these three samples are shown in Figure 3(b), Figure 3(c) and Figure 3(d)). The cycles are clearly rectangular, which means that the magnetizations of two ferromagnetic layers are turned over for the same magnetic field value. This indicates that the layers can be coupled FM. The FM coupling intensity is strong enough across the Ge layer so that the reversals of the magnetizations do not take place separately. In this type of coupling, the coercive field of the soft layer (low anisotropy) becomes higher while that of the hard layer decreases its initial value, ultimately resulting in a very small difference in the inversion field of the two layers. The results obtained from Figure 3 also show an increase in the saturation field Hs of the sample with $\mathrm{d}_{\mathrm{Ge}}=3 \mathrm{~nm}$ (about $1.10^{4} \mathrm{Oe}$ for $\mathrm{d}=1 \mathrm{~nm}$ and $15 \mathrm{~nm}$ while it is greater than $2.10^{4}$ Oe for $\mathrm{d}=3 \mathrm{~nm}$ ).

In Figure 4, an amazing trend that we can see in this series is a decrease in the coercive field $\mathrm{H}_{\mathrm{C}}$ down to 120 Oe for $\mathrm{d}=3 \mathrm{~nm}$ while in the other two cases, they are almost identical $\mathrm{H}_{\mathrm{C}} \approx 250$ Oe. A similar trend can also be observed for the remanent magnetization $\left(M_{r}\right)$. However, due to the small number of samples measured, we cannot say that the Hc field oscillates with the thickness of Ge. It is clear that the intensity of the exchange coupling is different for each thickness, confirming that the magnetic interactions or exchange coupling between the layers exist and depend on the thickness of the Ge spacers. For $d=1 \mathrm{~nm}$, the 

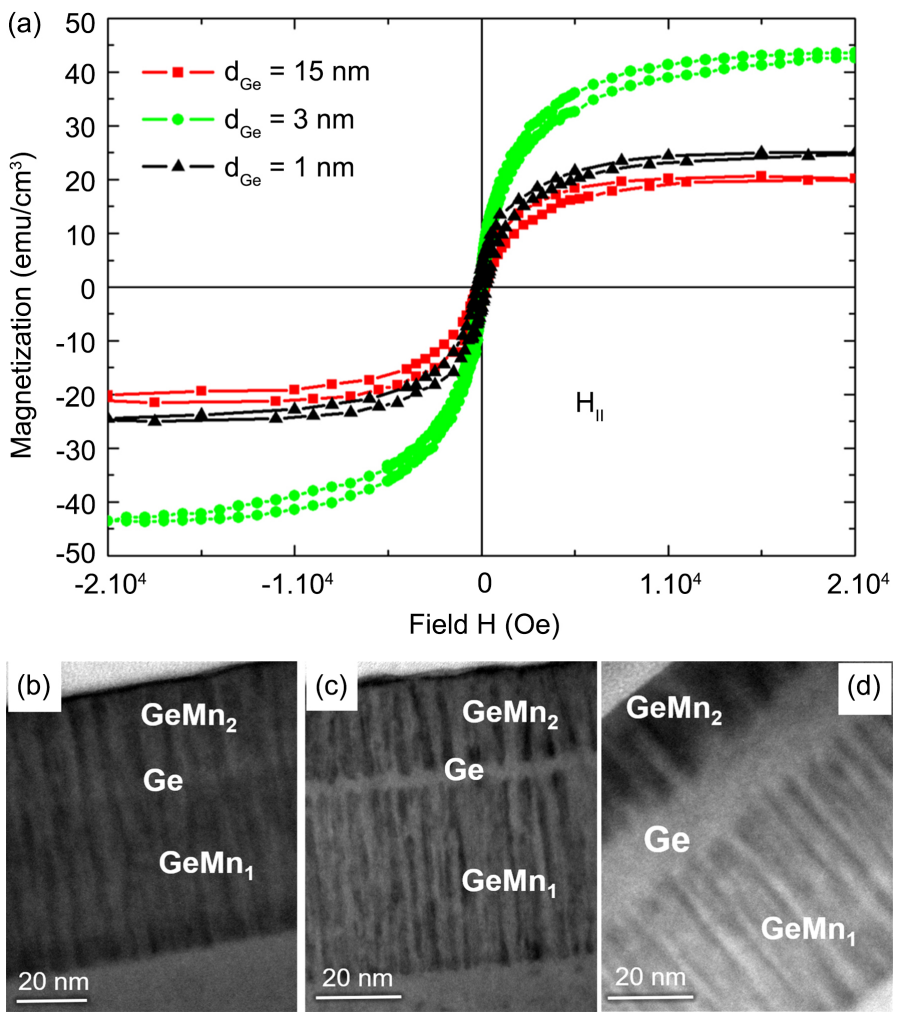

Figure 3. (a) Hysteresis loops measured at $10 \mathrm{~K}$ (a) and TEM images of three samples $\mathrm{Ge}_{0.94} \mathrm{Mn}_{0.06}(45 \mathrm{~nm}) / \mathrm{Ge}(\mathrm{d}) / \mathrm{Ge}_{0.94} \mathrm{Mn}_{0.06}(25 \mathrm{~nm})$ with $\mathrm{d}=1 \mathrm{~nm}(\mathrm{~b}), \mathrm{d}=3 \mathrm{~nm}(\mathrm{c})$ and $\mathrm{d}=15 \mathrm{~nm}(\mathrm{~d})$.

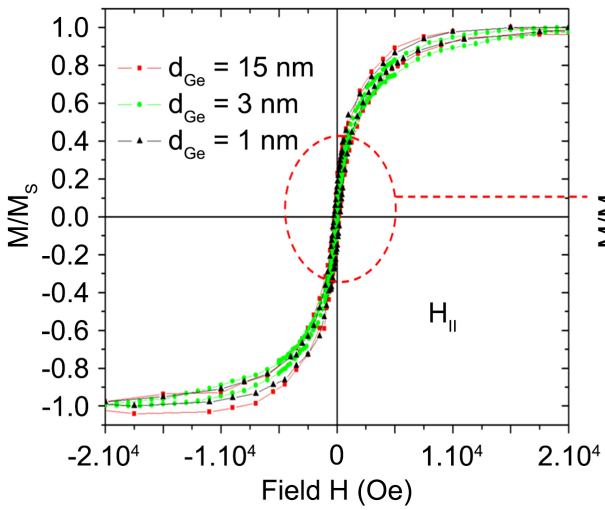

(a)

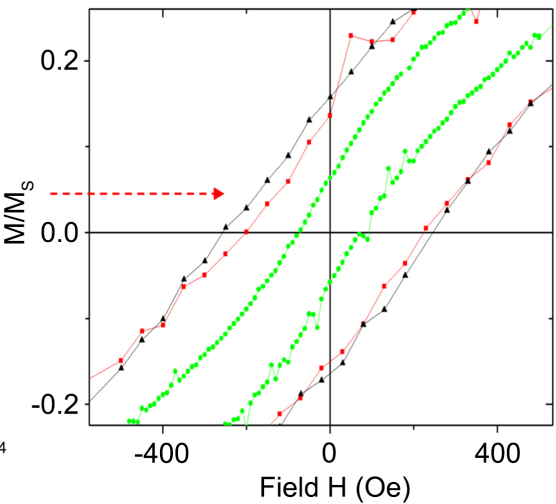

(b)

Figure 4. Normalization of hysteresis cycles in Figure 3(a) and the enlargement of the curves shows a clear decrease in $\mathrm{H}_{\mathrm{C}}$ for $\mathrm{d}_{\mathrm{Ge}}=3 \mathrm{~nm}$ compared to the two other thicknesses (b).

TEM images of this sample (Figure $3(\mathrm{~b})$ ) showed a very indistinct interface as we had a single layer of continuous nano columns. The coupling in this case must be important because the effect of the surface gives rise, in general, to a positive effect for FM coupling. With regard to $d=3 \mathrm{~nm}$, the attenuation of the coercive field is considerable and the field necessary to saturate the sample is greater, which means that the coupling length is significantly different compared 
to the case of $d=1 \mathrm{~nm}$. It is important to note that in the case of the perpendicular configuration (not show here), we measured the variation of the magnetization with the field for two samples $(\mathrm{d}=3 \mathrm{~nm}$ and $15 \mathrm{~nm})$ and the result does not reflect the same behavior: the values of $\mathrm{H}_{\mathrm{C}}$ and $\mathrm{H}_{\mathrm{S}}$ change only very slightly, which implies that the magnetic anisotropy of nanocolumns plays no role in the exchange coupling for this type of system.

We have just shown the structure of spin valves based on nanocolumns an attenuation of the FM coupling which means that there is a transition towards either a biquadratic coupling which seems most likely because of the anisotropy of the system, or AF coupling or total decoupling of the system. The exchange coupling problem for semiconductor spacers is not simple and in the multilayer system of nanocolumns, it seems even more complex, for the following reasons: 1) The leakage field induced by neighboring nanocolumns: magnetic coupling even in the case of a layer of nanocolumns is already complicated because the ferromagnetic layer is not a magnetically continuous medium, the magnetic nanocolumns couple together. To simplify the problem, we assume that nanocolumns in a layer are magnetic monodomains that have a preferred orientation. In multilayers, the contributions in exchange coupling are numerous and come from all the couplings between the nanocolumns of one layer and those of the two neighboring layers. The leakage field therefore becomes significant; 2) The magnetic anisotropy of nanocolumns: as have discussed in previous papers, nanocolumns have a fairly complex magnetic anisotropy and are considered to be a magnetically isotropic material, at least for both the parallel and perpendicular configurations [18] [21]. These magnetic properties make the phenomenon of magnetization reversal along the easy magnetization axes difficult.

\section{Conclusion}

In summary, we have studied the exchange coupling in the multilayer system GeMn nanocolumns/Ge in two configurations with the variation of Ge spacers. The results obtained show that, exchange coupling between the layers exists and depends on the Ge spacer thickness for the GeMn nanocolumns/Ge multilayer spin valve configuration. However, in order to have a more definitive conclusion and to determine the nature of the coupling, a study as a function of the Ge thickness accompanied by the measurement of minor cycles would be essential.

\section{Acknowledgements}

This work was supported by Hong Duc University under grant number of 564/HĐ-ĐHHĐ. The authors thank colleagues at Interdisciplinary Center of Nanoscience of Marseille (CINaM-CNRS), Aix-Marseille University, France for their technical helps and fruitful discussions.

\section{Conflicts of Interest}

The authors declare no conflicts of interest regarding the publication of this paper. 


\section{References}

[1] Baibich, M.N., Broto, J.M., Fert, A.F., Nguyen, V.D., Petroff, F., Etienne, P., Creuzet, G., Friederich, A. and Chazelas, J. (1988) Giant Magnetoresistance of $\mathrm{Fe}(001) / \mathrm{Cr}(001)$ Magnetic Superlattices. Physical Review Letters, 61, 2472-2475. https://doi.org/10.1103/PhysRevLett.61.2472

[2] Binasch, G., Grünberg, P., Saurenbach, F. and Zinn, W. (1989) Enhanced Magnetoresistance in Layered Magnetic Structures with Antiferromagnetic Interlayer Exchange. Physical Review B, 39, 4828. https://doi.org/10.1103/PhysRevB.39.4828

[3] Bruno, P. (1995) Theory of Interlayer Magnetic Coupling. Physical Review B, 52, 411. https://doi.org/10.1103/PhysRevB.52.411

[4] Slonczewski, J.C. (1989) Conductance and Exchange Coupling of Two Ferromagnets Separated by a Tunneling Barrier. Physical Review B, 39, 6995. https://doi.org/10.1103/PhysRevB.39.6995

[5] Stiles, M.D. (2005) Ultrathin Magnetic Structures III: Fundamentals of Nanomagnetism, Volume 3 (Interlayer Exchange Coupling). Springer, Berlin.

[6] Jungwirth, T., Atkinson, W.A., Lee, B.H. and MacDonald, A.H. (1999) Interlayer Coupling in Ferromagnetic Semiconductor Superlattices. Physical Review B, 59, 9818. https://doi.org/10.1103/PhysRevB.59.9818

[7] Strijkers, G.J., Kohlhepp, J.T., Swagten, H.J.M. and de Jonge, W.J.M. (2000) Origin of Biquadratic Exchange in Fe/Si/Fe. Physical Review Letters, 84, 1812.

https://doi.org/10.1103/PhysRevLett.84.1812

[8] Mattson, J.E., Kumar, Sudha, Fullerton, Eric, E., Lee, S.R., Sowers, C.H., Grimsditch, M., Bader, S.D. and Parker, F.T. (1993) Photoinduced Antiferromagnetic Interlayer Coupling in Fe/(Fe-Si) Superlattices. Physical Review Letters, 71, 185. https://doi.org/10.1103/PhysRevLett.71.185

[9] Yaacoub, N., Meny, C., Bengone, O. and Panissod, P. (2006) Short Period Magnetic Coupling Oscillations in Co/Si Multilayers: Theory versus Experiment. Physical Review Letters, 97, Article ID: 257206. https://doi.org/10.1103/PhysRevLett.97.257206

[10] Kepa, H., Kutner-Pielaszek, J., Blinowski, J., Twardowski, A., Majkrzak, C.F., Story, T., Kacman, P., Galazka, R.R., Ha, K., Swagtenand, H.J.M., de Jonge, W.J.M., Yu Sipatov, A., Volobuev, V. and Giebultowicz, T.M. (2001) Antiferromagnetic Interlayer Coupling in Ferromagnetic Semiconductor Eus/PbS(001) Superlattices. EPL (Europhysics Letters), 56, 54. https://doi.org/10.1209/epl/i2001-00487-7

[11] Gareev, R.R., Burgler, D.E., Buchmeier, M., Olligs, D., Schreiber, R. and Grunberg, P. (2001) Metallic-Type Oscillatory Interlayer Exchange Coupling across an Epitaxial FeSi Spacer. Physical Review Letters, 87, Article ID: 157202. https://doi.org/10.1103/PhysRevLett.87.157202

[12] Dau, M.T., Le Thanh, V., Le, T.G., Spiesser, A., Petit, M., Michez, L.A. and Daineche, R. (2011) Mn Segregation in Ge/ $\mathrm{Mn}_{5} \mathrm{Ge}_{3}$ Heterostructures: The Role of Surface Carbon Adsorption. Applied Physics Letters, 99, Article ID: 151908. https://doi.org/10.1063/1.3651488

[13] Dau, M.T., Le Thanh, V., Le, T.G., Spiesser, A., Petit, M., Michez, L., Le, T.G., Abbes, O. and Ranguis, A. (2012) An Unusual Phenomenon of Surface Reaction Observed during Ge Overgrowth on $\mathrm{Mn}_{5} \mathrm{Ge}_{3} / \mathrm{Ge}(111)$ Heterostructures. New Journal of Physics, 14, Article ID: 103020. https://doi.org/10.1088/1367-2630/14/10/103020

[14] Michez, L.A., Spiesser, A., Petit, M., Bertaina, S., Jacquot, J.F., Didier, D. and Le Thanh, V. (2015) Magnetic Reversal in $\mathrm{Mn}_{5} \mathrm{Ge}_{3}$ Thin Films: An Extensive Study. Journal of Physics. Condensed Matter, 27, Article ID: 266001. 
https://doi.org/10.1088/0953-8984/27/26/266001

[15] Assaf, E., Portavoce, A., Hoummada, K., Bertoglio, M. and Bertaina, S. (2017) High Curie Temperature $\mathrm{Mn}_{5} \mathrm{Ge}_{3}$ Thin Films Produced by Non-Diffusive Reaction. Applied Physics Letters, 110, Article ID: 072408. https://doi.org/10.1063/1.4976576

[16] Olive Mendez, S.F., Petit, M., Ranguis, A., Le Thanh, V. and Michez, L. (2018) From the Very First Stages of Mn Deposition on Ge(001) to Phase Segregation. Crystal Growth \& Design, 18, 5124-5129. https://doi.org/10.1021/acs.cgd.8b00558

[17] Kalvig, R., Jedryka, E., Wojcik, M., Petit, M. and Michez, L. (2020) Selective Modification of the Unquenched Orbital Moment of Manganese Introduced by Carbon Dopant in Epitaxial $\mathrm{Mn}_{5} \mathrm{Ge}_{3} \mathrm{C}_{0.2} / \mathrm{Ge}(111)$ Films. Physical Review B, 101, Article ID: 094401. https://doi.org/10.1103/PhysRevB.101.094401

[18] Le, T.G., Le Thanh, V. and Michez, L. (2020) Effect of Carbon on Structural and Magnetic Properties of $\mathrm{Ge}_{1-x} \mathrm{Mn}_{x}$ Nanocolumns. Bulletin of Materials Science, 43, 103. https://doi.org/10.1007/s12034-020-2082-z

[19] Le, T.G., Dau, M.T., Le Thanh, V., Nam, D.N.H., Petit, M., Michez, L. and Khiem, N.V. (2012) Growth Competition between Semiconducting $\mathrm{Ge}_{1-x} \mathrm{Mn}_{x}$ Nanocolumns and Metallic $\mathrm{Mn}_{5} \mathrm{Ge}_{3}$ Clusters. Advances in Natural Sciences. Nanoscience and Nanotechnology, 3, Article ID: 025007. https://doi.org/10.1088/2043-6262/3/2/025007

[20] Jamet, M., Barski, A., Devillers, T., Poydenot, V., Dujardin, R., Bayle-Guillemaud, P., Rothman, J., Bellet-Amalric, E., Marty, A. and Cibert, J. (2006) High-Curie-Temperature Ferromagnetism in Self-Organized $\mathrm{Ge}_{1-x} \mathrm{Mn}_{x}$ Nanocolumns. Nature Materials, 5, 653-659. https://doi.org/10.1038/nmat1686

[21] Devillers, T., Jamet, M., Barski, A., Poydenot, V., Bayle-Guillemaud, P., Bellet-Amalric, E., Cherifi, S. and Cibert, J. (2007) Structure and Magnetism of Self-Organized $\mathrm{Ge}_{1-x} \mathrm{Mn}_{x}$ Nanocolumns on Ge(001). Physical Review B, 76, Article ID: 205306. https://doi.org/10.1103/PhysRevB.76.205306

[22] Le, T.G. and Dau, M.T. (2016) Vertical Self-Organization of $\mathrm{Ge}_{1-x} \mathrm{Mn}_{x}$ Nanocolumn Multilayers Grown on Ge(001) Substrates. Modern Physics Letters B, 30, Article ID: 1650269. https://doi.org/10.1142/S0217984916502699

[23] Le, T.G. (2015) Direct Structural Evidences of Epitaxial Growth $\mathrm{Ge}_{1-x} \mathrm{Mn}_{x}$ Nanocolumn Bi-Layers on $\mathrm{Ge}(001)$. Materials Sciences and Applications, 6, 533-538.

http://www.scirp.org/journal/msa https://doi.org/10.4236/msa.2015.66057

[24] Tardif, S., Cherifi, S., Jamet, M., Devillers, T., Barski, A., Schmitz, D., Darowski, N., Thakur, P., Cezar, J.C., Brookes, N.B., Mattana, R. and Cibert, J. (2010) Exchange Bias in GeMn Nanocolumns: The Role of Surface Oxidation. Applied Physics Letters, 97, Article ID: 062510. https://doi.org/10.1063/1.3476343 